\title{
Je schwerer die Psoriasis, desto höher das Diabetesrisiko
}

Patienten mit Psoriasis haben ein höheres Risiko, an Diabetes mellitus zu erkranken. Laut Ergebnissen einer Studie aus Taiwan bleibt die Zunahme der Inzidenz auch nach Abgleich gegen Begleitkrankheiten und Medikation erhalten.

Verglichen mit der Allgemeinpopulation sind Prävalenz und Inzidenz von Diabetes unter Psoriasispatienten erhöht. Wie dabei die krankheitsbedingten chronischen Entzündungsprozesse, begleitende Erkrankungen und die Effekte der in der Therapie eingesetzten Medikamente zusammenwirken, wollten Dermatologen um MengSui Lee (Taipeh) herausfinden. Zu diesem Zweck wurden rund 7000 Patienten, deren Psoriasis zwischen 1999 und 2008 diagnostiziert worden war, ebenso vielen gesunden Kontrollpersonen gegenübergestellt.

Registriert wurden neu auftreten-

Quelle:

springermedizin.de, basierend auf: Meng-Sui L et al. Increased risk of diabetes mellitus in relation to the severity of psoriasis, concomitant medication, and comorbidity: A nationwide population-based cohort study. J Am Acad Dermatol 2014, online 10 Januar; doi: 10.1016/j.jaad.2013.11.023 de Fälle von Typ-2-Diabetes. Die Diabetesinzidenz war in der Psoriasiskohorte signifikant erhöht. Sie betrug bei mildem Verlauf 10,5, bei schwerer Erkrankung 18,2 pro 1000 Personenjahre, verglichen mit 5,4/1000 bei den Kontrollen. Die kumulativen Ein-, Fünf- und AchtJahres-Inzidenzen betrugen 3,1\%, 8,8\% und $14 \%$ bei schweren und $1,2 \%, 4,9 \%$ und $8,3 \%$ bei leichten Formen (Kontrollen: $0,8 \%, 2,6 \%, 4,3 \%)$.

\section{Einfluss der einzelnen Faktoren} Hernach kalkulierten die taiwanischen Forscher den Einfluss verschiedener Faktoren unter Abgleich nach den jeweils übrigen relevanten Parametern. Daraus ergab sich, immer im Vergleich zur Kontrollgruppe, folgendes Bild:

- Psoriasis insgesamt schlug mit einer Risikosteigerung für Typ-2Diabetes von $35 \%$ zu Buche (Hazard Ratio $[H R] 1,35)$ schwere Psoriasis verdoppelte die Inzidenz (HR 2,06), leichtere Formen erhöhten sie um $28 \%$ (HR 1,28).

- Jedes zusätzliche Lebensjahr ließ das Diabetesrisiko um $3 \%$ steigen.

- Die Steigerungfaktoren für verschiedene Begleiterkrankungen betrugen 3,13 für Dyslipidämie, 2,82 für Hypertonie und 2,01 für Morbus Cushing.

- Pro 100 Psoralen-plus-UV-A(PUVA)-Behandlungen lag die Inzidenz 2,58-fach höher.

- Je 100 Tagesdosen Methotrexat innerhalb von zehn Monaten stieg das Risiko um 20\%.
- 100 Dosen eines Thiazids binnen eines Jahres steigerten die Diabetesinzidenz um $17 \%$.

\section{Medikamente und Diabetesrisiko}

Biologika waren laut den Autoren bis Ende 2009 in Taiwan nicht für die Psoriasistherapie zugelassen und gingen daher nicht in die Analyse ein.

„Diese Ergebnisse haben gezeigt, dass Patienten mit Psoriasis ein mäBig erhöhtes Risiko tragen, an Diabetes mellitus Typ 2 zu erkranken“, schreiben Lee und Kollegen. Das Risiko sei durch die Krankheitsschwere, Komorbidität und dosisabhängig durch die begleitende Medikation modifiziert worden. „Psoriasispatienten und ihre Ärzte sollten sich der möglichen Verbindung zwischen den verordneten Medikamenten und Diabetes bewusst sein", so das Fazit der Wissenschaftler.

hautnah 2015 · 14:8

DOI 10.1007/s12326-014-0136-y Online publiziert: 22. Oktober 2014 (c) Springer-Verlag Wien 2014 